\section{THU0615 IMMUNE RELATED ADVERSE EVENTS (IRAES ASSOCIATED WITH CHECKPOINT INHIBITORS: 12 CASES FROM A SINGLE CENTRE}

D. Ennis, F. To, S. Jamal. Rheumatology, University of British Columbia, Vancouver, Canada

Background: Immune checkpoint inhibitors (ICI) have made a significant impact on the treatment of many advanced malignancies. There is little data on the rheumatologic complications of these treatments.

Objectives: We describe 12 cases of rheumatologic IRAEs following ICI treatment to further characterise the spectrum of disease and treatment responses. Methods: We report patients evaluated in a general Rheumatology outpatient clinic from 2014 to 2017. Cases were defined as those with new rheumatologic symptoms following treatment with an ICl. Alternative explanations for the presenting syndrome were excluded clinically. Clinical data was extracted by retrospective chart review.

Results: This case series includes 12 patients ( 6 female, 6 male) with a mean age at IRAE onset of 63.9 years (range 33-79). Multiple cancers were represented including melanoma $(n=9)$, Hodgkin's lymphoma $(n=1)$, squamous cell lung cancer $(n=1)$, and adenocarcinoma of the lung $(n=1) .5 / 12$ patients received Nivolumab, 8/12 received Pembrolizumab, and 2/9 received Ipilimumab. ICl exposure was associated with various rheumatologic IRAEs including PMR-like syndrome $(n=4)$, symmetric polyarthritis $(n=6)$, psoriatic arthritis $(n=1)$, oligoarthritis $(n=1)$, and erythema nodosum $(n=1)$. Other IRAEs were also noted including vitiligo $(n=1)$, pulmonary capillaritis $(n=1)$, ulcerative colitis flare $(n=1)$, inflammatory seborrheic keratosis and psoriasis $(n=1)$. The mean time of onset of the IRAE from the first exposure to an $\mathrm{ICl}$ was 6.8 months (range 0-21 months). In 7 cases, rheumatologic symptoms worsened with each $\mathrm{ICl}$ dose. Laboratory investigations demonstrated elevated CRP in 7 cases (mean 75.6; range 3.7-290.1), RF positivity in 2 cases, weak positive ANAs in 4 cases (1:80), SSA positivity in 2 cases, and a single case where a pre-existing anti-CCP antibody was identified. Steroids were used in 11 cases at a mean starting dose of $36 \mathrm{mg}$ (range 10-50 mg) by mouth daily for an average duration of 6.1 months (range 1-12 months). Other DMARDs were necessary in some cases (Hydroxychloroquine $n=1$; Methotrexate $\mathrm{n}=5$ ). While 6 patients experienced rapid improvement, 4 experienced gradual improvement. Most patients achieved partial resolution of symptoms $(n=6)$ while only 4 achieved complete resolution. Tumour response was observed in all 12 patients.

Conclusions: This case series of IRAEs associated with ICI treatment suggest that symmetric polyarthritis and PMR-like syndromes are the most common rheumatologic IRAEs, although the spectrum is broad. IRAEs seem to develop around 6 months after first exposure, worsen with ongoing doses of $\mathrm{ICI}$ administration, and respond to treatment with corticosteroids. Treatment doses and duration were higher than expected for phenotype, with few patients achieving significant improvement with short courses. Those with IRAEs tend to have good tumour response, despite concurrent use of immunosuppressants. MTX and HCQ appear to be safe and effective, but more experience with these and other DMARDs/biologics is required in these patients.

Disclosure of Interest: None declared

DOI: 10.1136/annrheumdis-2018-eular.6009

\section{THU0616 ELEVATED THYROID STIMULATING HORMONE AS A POTENTIAL BIOMARKER FOR RHEUMATIC IMMUNE- RELATED ADVERSE EVENTS FOLLOWING PD-1 INHIBITOR THERAPY}

D.F.L. Liew ${ }^{1,2,3}$, J.L.Y. Leung ${ }^{1,3}$, C. McMaster ${ }^{2}$, F. Djukiadmodjo ${ }^{2}$, B. Liu ${ }^{1}$, J. Cebon ${ }^{4}$, A.G. Frauman ${ }^{2,3}$, R.R.C. Buchanan ${ }^{1,3} .{ }^{1}$ Rheumatology, ${ }^{2}$ Clinical Pharmacology and Therapeutics, Austin Health, Heidelberg; ${ }^{3}$ Medicine, University of Melbourne, Parkville: ${ }^{4}$ Olivia Newton-John Cancer Wellness and Research Centre, Heidelberg, Australia

Background: Programmed cell death protein (PD-1) inhibitor immunotherapy is being increasingly used in oncology, but may cause immune-related adverse events (irAEs) resembling classical rheumatic and non-rheumatic autoimmune diseases. While oncological response to therapy has been associated with the development of rheumatic irAEs, no biomarker to predict the development of rheumatic irAEs has yet been identified. Thyroid stimulating hormone (TSH) is readily available in clinical practice and is frequently tested prior to the administration of PD-1 inhibitors to screen for existing thyroid disease.

Objectives: To investigate whether thyroid stimulating hormone, or changes to it, are associated with the development of immune-related adverse events (irAEs) following PD-1 inhibitor therapy for cancer.

Methods: This was a retrospective chart review of all patients at a single centre who had a TSH level performed in the institutional laboratory prior to the patient being dispensed nivolumab or pembrolizumab before January 1, 2017, with follow-up until July 1, 2017. TSH levels before and during PD-1 inhibitor therapy were recorded. Patients with any diagnosis of a rheumatic irAE or any non- cutaneous irAE were identified. Linear regression was performed to determine the relationship between TSH and the development of irAEs. Youden's index was used to derive the optimal cut-off point. Recursive partitioning methods with imbalanced prior probabilities were used to create a decision tree model.

Results: There were 213 episodes of therapy which met criteria, of which a noncutaneous irAE occurred in 62 episodes $(29.1 \%)$, thyroid irAEs occurred in 22 episodes $(10.3 \%)$ and rheumatic irAEs were diagnosed in 16 episodes (7.5\%). Even when corrected for duration of exposure to PD-1 inhibitor therapy, elevated TSH levels (>4.2 $\mathrm{mU} / \mathrm{L}$ ) were statistically significantly associated with the development of rheumatic irAEs (adjusted OR 6.08,1.53-24.22), and this was not weakened by excluding patients who went on to develop thyroid irAEs. There was no significant association between elevated TSH levels and the development of any noncutaneous irAE. Change in TSH levels with PD-1 inhibitor therapy was also not associated with the development of non-cutaneous irAEs or rheumatic irAEs specifically. Using a TSH level $>2.4$ to predict rheumatic irAEs led to a positive predictive value of $25 \%$ and a negative predictive value of $93 \%$ in our cohort. A decision tree model to predict rheumatic irAEs combining a pre-PD-1 inhibitor TSH level $>2.4$ and an oncological response to therapy led to a positive predictive value of $50 \%$ and a negative predictive value of $94 \%$ in our cohort.

Conclusions: Elevated TSH levels may be a potential biomarker for the development of rheumatic irAEs. In particular, a pre-PD-1 inhibitor TSH level of $>2.4 \mathrm{mU} /$ $\mathrm{L}$, in combination with oncological response to therapy, may identify patients at risk of rheumatic irAEs. Associations observed in this cohort should be examined in larger cohorts to determine the clinical utility of TSH in predicting rheumatic irAEs.

Disclosure of Interest: None declared

DOI: 10.1136/annrheumdis-2018-eular.6653

\section{THU0617 IS THE NUMBER OF IGG4+ PLASMA CELLS SEEN BY IMMUNOSTAINING USEFUL BEYOND ITS DIAGNOSTIC UTILITY IN IGG4-RELATED DISEASE?}

E. Martin Nares ${ }^{1}$, J. Guerrero Castillo², A. Angeles Angeles ${ }^{2}$, G. Hernández Molina ${ }^{1} .{ }^{1}$ Immunology and Rheumatology Department, ${ }^{2}$ Pathology Department, Instituto Nacional de Ciencias Médicas y Nutrición Salvador Zubirán, Mexico City, Mexico

Background: The histopathological findings in IgG4-related disease (IgG4-RD) includes the presence of marked lgG4 + plasma cell infiltration seen by immunostaining and it has been used in clinical practice only as a diagnostic tool. Whether the number of $\lg G 4$ + plasma cells in tissue is associated with any clinical or serological feature of the disease has not been previously evaluated.

Objectives: To evaluate if the number of lgG4 +plasma cell infiltration is associated with any clinical or serological outcome.

Methods: We included 30 patients with biopsy proven IgG4-RD according to the Comprehensive Diagnostic Criteria for IgG4-RD who regularly attended a tertiary referral centre in Mexico City (2000-2017). We collected demographics, clinical (organ involvement, relapses and the disease activity assessed by the IgG4-RD Responder Index [lgG4-RD RI] at baseline) as well as baseline laboratory data (C3, C4, total eosinophil count, IgG4 levels). Patients were divided in three groups according to the number of IgG4 + plasma cells seen by immunostaining as follows: $<50 \mathrm{lgG} 4$ +plasma cells/HPF, 50-100 lgG4 +plasma cells/HPF, and >100 lgG4 + plasma cells/HPF.

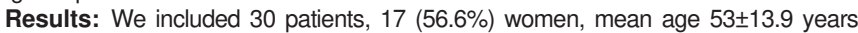
and median disease duration 13 months. The biopsies were from the following tissues: lacrimal gland $(n=6)$, pancreas $(n=5)$, orbit $(n=4)$, kidney $(n=4)$, lymph node $(n=3)$, mediastinum $(n=2)$, salivary gland $(n=2)$ and other tissues $(n=4)$. Eleven patients $(36.6 \%)$ had $<50$ lgG4 +plasma cells/HPF, 9 patients (30\%) 50-100 lgG4 +plasma cells/HPF and 10 (33.3\%) patients $>100$ lgG4 +plasma cells/HPF. We did not find any difference regarding age, gender, time of follow up, number of involved organs and relapses. The median basal lgG4-RD RI was 9,6 and 15, for the $<50$ lgG4 +plasma cells/HPF, 50-100 lgG4 +plasma cells/HPF, and $>100$ $\operatorname{lgG} 4$ +plasma cells/HPF groups respectively, however, they did not reach statistica significance. The group with $>100 \mathrm{lgG} 4$ + plasma cells/HPF had more frequently lymphadenopathy when compared with the other groups $(36.4 \%, 66.7 \%$ and $80 \%$, $\mathrm{p}=0.02$; respectively) while the proportion of involvement of the other anatomic sites were similar. We found a statistical difference in serum C3 levels $(99.5 \mathrm{mg} / \mathrm{dl}$, $159 \mathrm{mg} / \mathrm{dl}, 78.5 \mathrm{mg} / \mathrm{dl}, \mathrm{p}=0.04)$ and a tendency for serum C4 levels $(20 \mathrm{mg} / \mathrm{dl}$, $27 \mathrm{mg} / \mathrm{dl}$, and $6 \mathrm{mg} / \mathrm{dl}, \mathrm{p}=0.08$ ) among the groups, whereas the levels of serum lgG4 $(60.8 \mathrm{mg} / \mathrm{dl}, 196 \mathrm{mg} / \mathrm{dll}, 245 \mathrm{mg} / \mathrm{dl}, \mathrm{p}=0.35)$ and the eosinophil count $\left(284 / \mathrm{mm}^{3}, 189 /\right.$ $\mathrm{mm}^{3}, 283 / \mathrm{mm}^{3}, p=0.46$ ) were similar. The $\mathrm{C} 3$ and $\mathrm{C} 4$ serum levels negatively correlated with the basal lgG4-RD RI ( $\tau=-0.48, p=0.005$ and $\tau=-0.58, p=0.001)$.

Conclusions: Our results show that the number of lgG4 +plasma cells seen by immunostaining in IgG4-RD may be of value in identifying a subset of patients with hypocomplementemia, lymphadenopathy and probably higher basal disease activity. The finding of an association between hypocomplementemia and higher tissue infiltration by $\lg G 4$ +plasma cells expands the evidence that complement activation may contribute to the pathogenesis of IgG4-RD. 